\title{
Response to Martini and Habeck: Semiochemical Dose-response Curves Fit by Kinetic Formation Functions
}

\author{
John A. Byers
}

Published online: 12 December 2014

(C) Springer Science+Business Media New York (outside the USA) 2014

Martini and Habeck (2014) correctly describe the conceptual simulation model of Byers (2013) where molecules in an odor filament pass by an antenna causing an electrophysiological antennographic (EAG) response that is proportional to how many of the receptors are hit at least once by a molecule. Increasing the doses (numbers) of molecules would cause increasing numbers of depolarized receptors that result in a dose-EAG response curve. Byers (2013) used non-linear regression software (TableCurve 2D version 5.01, Systat Software Inc., Chicago, IL, USA) to fit many functions including the logarithmic $\left(r^{2}=0.773\right)$ to the simulated data. However, kinetic formation functions fit the simulated data best $\left(\mathrm{r}^{2}=\right.$ 0.99999), as well as many dose-response data sets in the literature. A family of kinetic formation functions was favored because they describe chemical reaction rates, which are known to be catalyzed by complex chemical binding and enzymatic processes in the antenna and central nervous system (Sachse and Krieger, 2011).

Martini and Habeck (2014) developed a probabilistic model derived from the simulation model of Byers (2013). Their equation (2) gives the expected number of receptors hit at least once, where $N=$ number of receptors, $n=$ number of molecules, and $m=$ number of antennal areas that can receive hits by molecules, to give the mean results of each simulated value shown in the first-order kinetic formation regression of Fig. 3 in Byers (2013). For example, $N=10^{4}, n=10^{6}$, and $m=10^{6}$ would give 6,321 receptors depolarized. Martini and Habeck also derived an equation for the variance, (e.g., 2,312 or SD = 48 for the above parameters).

This reply refers to the comment available at doi:10.1007/s10886-0140526-x.

J. A. Byers $(\bowtie)$

US Arid-Land Agricultural Research Center, USDA-ARS,

Maricopa, Arizona, USA

e-mail: john.byers@ars.usda.gov

Martini and Habeck (2014) confirm that a first-order kinetic formation function is consistent with the simulation model of Byers (2013) where odor molecules are "shot" independently at the antenna with the "assumption that they move only in one direction". While Byers described molecules moving in one direction, the molecules could come from any direction as long as $n$ molecules hit the antenna. Martini and Habeck suggest the possibility that equilibrium equations may be more adequate because of odorant-binding proteins (OBPs) involved in the dose-response process at the antennal level. Besides OBPs, there are trans-membrane enzyme complexes, odor degrading enzymes, and interneuronal connections that would probably add complexity to the kinetics (Sachse and Krieger, 2011). Although kinetic formation functions of first to third order and variable order (Table 1 in Byers 2013) fit many dose-response data sets in the literature, kinetic functions also include formation-equilibrium ones that may fit as well or better to various dose-response data sets. Among the kinetic equilibrium models in TableCurve 2D, three functions with three, four, or six terms fit as well as the first-order kinetic formation function. The simple equilibrium function with three terms, $Y=(a-b) \exp (-c X)+b$, fit the simulation results $\left(\mathrm{r}^{2}=0.99999\right)$ when $a=0.88826, b=9999.3$, and $c=$ 0.000001002 . For most chemical ecologists, using regression software will be the most convenient method of fitting a doseresponse curve, and the family of kinetic formation functions (including equilibrium ones) appear to have a theoretical basis.

My purpose was to gain insight through a simple model that reflected our general understanding of the natural process of EAG and dose-responses. Functions that describe the relationship between dose and response will fit less well when there are more interacting processes causing increased complexity and variation. For example, in behavioral bioassays in the laboratory, the antenna receives the semiochemical molecules that give an EAG, but additional steps are involved in order to produce a behavioral response. Still 
poorer fits for dose-response curves in the field could be expected due to increased variability from the combined EAG, behavioral, and environmental variation. This appears to be the case. Analysis of 45 EAG studies in the literature (Byers 2013) showed the kinetic formation functions (A-L) had a mean adjusted $r^{2}$ of $0.966 \pm 0.062$ $( \pm \mathrm{SD})$. Laboratory bioassays in 23 experiments had a lower fit with A-L functions having an average $r^{2}=$ $0.912 \pm 0.149$. In the field, the fit of dose-response curves by kinetic formation functions was least good with an average $r^{2}=0.771 \pm 0.270$. Therefore, specific kinetic functions will fit certain dose-response curves better than others and may describe the effects of underlying mechanisms. However, molecular kinetic studies regarding the antenna and neurons in a species may ultimately provide the mechanistic basis for an appropriate dose-response function.

\section{References}

Byers JA (2013) Modeling and regression analysis of semiochemical dose-response curves of insect antennal reception and behavior. $\mathrm{J}$ Chem Ecol 39:1081-1089

Martini JWR, Habeck M (2014) Kinetics or equilibrium? - A commentary on the recent simulation study of semiochemical dose-response curves of insect olfactory sensing. J Chem Ecol, this volume.

Sachse S, Krieger J (2011) Olfaction in insects: The primary processes of odor recognition and coding. E-neuroforum 2:49-60 